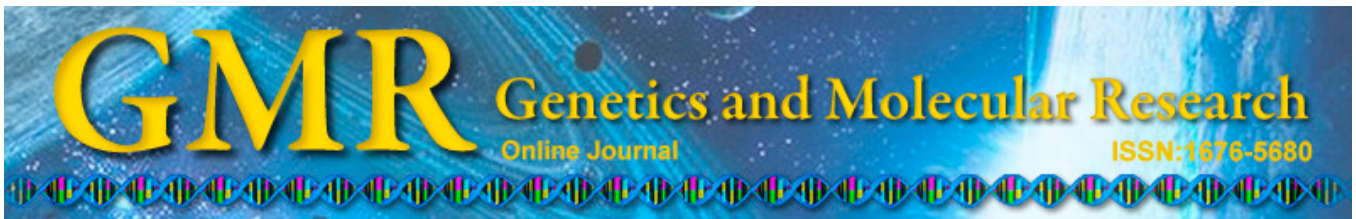

\title{
Diversity resistance to Puccinia striiformis f. sp Tritici in rye chromosome arm 1RS expressed in wheat
}

\author{
M.Y. Yang, T.H. Ren, B.J. Yan, Z. Li and Z.L. Ren \\ Key Laboratory for Plant Genetics and Breeding, \\ Sichuan Agricultural University, Chengdu, Sichuan, China \\ Corresponding authors: T.H. Ren / Z.L. Ren \\ E-mail: renxx@umn.edu / renzllab@sicau.edu.cn
}

Genet. Mol. Res. 13 (4): 8783-8793 (2014)

Received October 9, 2013

Accepted May 8, 2014

Published October 27, 2014

DOI http://dx.doi.org/10.4238/2014.October.27.20

\begin{abstract}
The 1BL.1RS wheat-rye translocation contained in the Russian cultivar Aurora has been the most widespread alien translocation in wheat-breeding programs all over the world. However, following the prevalence of new biotypes of the pathogens, disease-resistance genes in this translocation chromosome have been overcome and consequently they have been eliminated in modern wheat-breeding programs. In this paper, we report on 12 new primary 1BL.1RS translocation lines derived from the crosses of a Chinese high yield wheat cv. Mianyang 11 with three rye cultivars collected from China. GISH, C-banding and PCR techniques using the specific primers for 1BS, $1 \mathrm{RS}$ and centromeres of wheat and rye were applied to identify the constitution of chromosomes. The results confirmed that all 1BL.1RS chromosomes in the 12 primary translocation lines contained integrated 1RS chromosome arms. In the resistance analysis using five kinds of Pst pathotypes, the 12 primary translocation lines showed diversity resistance to stripe rust, which contained at least five different new genes (alleles), significantly different from the $Y r 9$ gene coming from Russian wheat cultivar Aurora. The results indicated that the chromosome arm 1RS in the rye population carries abundant yet untapped genes (alleles) for resistance to wheat stripe rust, which
\end{abstract}


would originate from the neutral diversity in the natural population of rye. It is suggested that creating more primary translocation lines in genome modification will be extremely important to use the diversity of alien R-genes, which was generated by long-term neutral mutation and maintained in the population of alien species.

Key words: Triticum aestivum; Secale cereale; 1BL.1RS translocation; Puccinia striiformis f. sp Tritici pathotypes; Diversity of R-genes

\section{INTRODUCTION}

In the past decade, great progress has been made related to the genes involved in plant resistance to pathogens. Many studies on their identification, mapping, and sequencing have allowed the elucidation of the genetic basis and different evolutionary processes of the R-genes (Liu et al., 2007; Salvaudon et al., 2008; Zhang et al., 2013). In plant-pathogen interactions there has been much emphasis on the "gene-for-gene" mechanism of resistance (Flor, 1971). Thus, there are very important questions of how much of the observed resistance diversity in host populations originated from selection by the interactions between host and parasites, and would nonselective events related to neutral evolution also offer diversity to R-genes?

Wheat stripe rust, caused by Puccinia striiformis f. sp Tritici (Pst), is considered one of the most devastating diseases around the world (Milus et al., 2009). Yield reduction caused by stripe rust can be as high as $75 \%$ in severe epidemics (Sharma-Poudyal and Chen, 2011). Breeding resistant cultivars is regarded as the most effective, economical and environmental method to control the disease. A gene against stripe rust, $\operatorname{Yr} 9$, located on the chromosome arm 1RS of rye (Secale cereale L.) cv. Petkus, has been the most successful and widespread application in wheat-breeding programs via a 1BL.1RS translocation from the Russian wheat cultivar Aurora and its derivates (Zeller and Fuchs, 1983; Zeller and Hsam, 1983; Berzonsky et al., 1991; Porter et al., 1993). According to Rabinovich (1998), several hundreds of wheat cultivars carrying this 1BL.1RS translocation have been released and the global cultivated area of these wheat cultivars occupies over five million hectares (Villareal et al., 1998). In China, approximately $70 \%$ of wheat cultivars in southwestern and $55 \%$ in northern regions carry the $Y r 9$ gene coming from the Russian wheat cultivars Aurora and Kavkaz and their derivates from the 1980s to the 1990s (Yang and Ren, 1997; Zhou et al., 2004).

Since the 1980s, $\operatorname{Yr} 9$-resistance genes located on 1BL.1RS have been overcome by new biotypes of pathogens (Zeller and Hsam, 1983; Lutz et al., 1992; Yang and Ren 1997, 2001; Shi et al., 2001). Unfortunately, the frequency of 1BL.1RS translocation in recently released wheat cultivars has declined (Ren et al., 2009). The key to the problem lies in a narrow genetic base contributed by the $1 \mathrm{RS}$ arm from cv. Petkus rye in most commercial translocation wheats (Villareal et al., 1998; Lelley et al., 2004). So far, according to previous research, only a few sources of $1 \mathrm{RS}$ were the progenitors of 1BL.1RS translocation in wheat worldwide. Ren et al. (2011a) considered that two were developed in Germany in the 1920s and 1930s, which derived from the rye source cv. Petkus, one bred from an octaploid triticale in Japan in the 1960s, others in the U.S., and one with rye cv. Paldanghomil in Korea. A 1AL.1RS translocation cultivar, Amigo, which is derived from the 1RS arm of rye cultivar Insave, has been a source in some North American wheat cultivars due to its resistance against powdery mildew, greenbug, stem rust, and wheat curl mite (Zeller and Fuchs, 1983; Zeller and Hsam, 1983; Con- 
ner et al., 1991; Rabinovich, 1998; Mater et al., 2004). Another 1AL.1RS translocation was selected in the 1990s (Porter et al., 1991, 1994). The two 1DL.1RS translocations were derived from the rye Imperial and Turkey 77, respectively (Shepherd, 1973; Marais et al., 1994).

Ren et al. (2009) put forward the view that it is crucial to introduce a substantial number of new genetic variations from other diploid rye sources into 1RS of the 1BL.1RS translocation, for a more efficient use of $1 \mathrm{RS}$ in wheat-breeding programs.

This study describes 12 new primary 1BL.1RS translocation lines, which were created from the cross of the wheat cultivar Mianyang11 (MY11) and three rye cultivars collected from China, and showed different resistance from translocation lines derived from Aurora. In this paper, we discuss the origination of diversity of R-genes in the rye population and suggest that genome modification by creating more primary translocation lines will be extremely valuable for the use of alien R-genes in wheat breeding in the future.

\section{MATERIAL AND METHODS}

\section{Plant materials}

The Chinese wheat cultivar MY11 (hexaploid, $2 \mathrm{~N}=\mathrm{AABBDD}=42$ ) as the pollen recipient, was crossed with three Chinese local rye varieties Baili, Weining, and Aigan (diploid, $2 \mathrm{~N}=\mathrm{RR}=14$ ) to obtain wheat $\mathrm{x}$ rye $\mathrm{F}_{1}$ hybrids, which were soaked in $0.05 \%$ colchicine $+3 \%$ dimethyl sulfoxide for $8 \mathrm{~h}$ to produce the amphidiploid $\left(\mathrm{C}_{1}\right)$. Since the rye chromosomes were rapidly eliminated in the process of backcrossing, $\mathrm{C}_{1}$ populations were backcrossed once or twice using the parental wheat cultivar MY11 for producing $1 \mathrm{R}$ monosomic wheat/rye addition lines. $1 \mathrm{R}$ monosomic addition lines were then propagated by selfing, to produce primary 1BL.1RS translocation lines (Ren et al., 1990b; Ren and Zhang, 1997). MY11, a high-yield and widely grown wheat cultivar in southwestern China, contains no rye chromatin and is highly susceptible to the Pst pathotypes used in the present study. Seeds of MY11 used in our study were produced through inbreeding with bagging for several generations to obtain pure genetic groups. The two rye varieties Weining and Baili originate from southwestern China, and Aigan was collected from northwestern China. These rye varieties are outcrossing populations and presumed to be genetically variable with respect to their $1 \mathrm{RS}$ chromosomes.

In addition, four 1BL.1RS translocation wheat cultivars were used as controls in cytogenetic identification, Chuannong10 (CN10), Chuannong11 (CN11), Chuannong17 (CN17) and Chuannong18 (CN18). The 1BL.1RS translocation of CN10 and CN11 was inherited from the Russian wheat cultivar Aurora, which contains the gene $Y r 9$ (Ren et al., 2009).

\section{Cytological analysis}

Chromosomes were treated with acid and alkali, where euchromatin DNA is easily digested but heterochromatin DNA is not due to its highly condensed nature. After Giemsa staining, chromosomes showed different bands, allowing their identification based on banding pattern. In this study, all materials were identified by Giemsa C-banding to determine the chromosome constitutions.

Furthermore, all 1BL.1RS translocation lines selected for this experiment were analyzed to verify the absence of any other detectable segment of rye by genomic in situ hybridization (GISH) (Lukaszewski et al., 2005). Rye genomic DNA was labeled with 
digoxigenin-11-dUTP as probe, and the rye signals were detected with fluorescein-conjugated anti-digoxigenin antibody, which gave yellow-green signals. Wheat chromosomes were stained with propidium iodide, which gave red signals.

\section{DNA extraction and polymerase chain reaction (PCR) analysis}

Total genomic DNA was isolated from young leaves according to Anderson et al. (1992). To confirm that the materials listed in Table 1 are 1BL.1RS translocations, two sets of primer pairs were used. One primer pair was O11B3 and O11B5 from the Glu-B3 gene on 1BS (Van Campenhout et al., 1995). The other primer pair was $\omega$-sec-P1 and $\omega$-sec-P2 (Chai et al., 2006). Four specific primers, O11B3 and O11B5, $\omega$-sec-P1 and $\omega$-sec-P2, were used together for DNA PCR (Chai et al., 2006). Primer pair PrCEN-2 (5'-AATG ATCT TCCA CGAC GACG-3', 5'-CCTC GTTG GGAA ATGG TGCA-3') was designed according to the nucleotides 1140-2090 of the pAWRC.1 sequence (GenBank accession No. AF245032). PrCEN-2 was used to produce a rye-specific centromeric sequence and to analyze the structure of the centromere of the 1BL.1RS chromosomes. PCR was carried out in a Bio-Rad icycler thermal cycler. DNA was amplified with $1 \mathrm{U}$ Taq DNA polymerase enzyme, $1 \mathrm{X}$ buffer, $1.5 \mathrm{mM} \mathrm{MgCl}$, $200 \mu \mathrm{M}$ dNTPs, $65 \mathrm{ng}$ primer, and $50 \mathrm{ng}$ DNA $/ 25 \mu \mathrm{L}$. After initial denaturation for 4 min at $94^{\circ} \mathrm{C}$, each cycle included $1 \mathrm{~min}$ denaturation at $94^{\circ} \mathrm{C}, 1 \mathrm{~min}$ annealing at $60^{\circ} \mathrm{C}$, and $1 \mathrm{~min}$ extension at $72^{\circ} \mathrm{C}$. A final extension for $10 \mathrm{~min}$ at $72^{\circ} \mathrm{C}$ followed the 30 cycles. The products of PCR amplification were separated on a $2 \%$ agarose gel.

Table 1. Responses to different Pst pathotypes among 12 1BL.1RS translocation lines originating from pure lines of wheat as recipients and three Chinese local rye varieties as donors.

\begin{tabular}{llccccccc}
\hline Line & Rye source & CYR25 & CYR29 & CYR30 & CYR31 & CYR32 & Yr gene & Phenotype \\
\hline RT1217-2 & Baili & 0 & 7 & 0 & 0 & 1 & $?$ & $?$ \\
RT1235-1 & Baili & 0 & 0 & 0 & 0 & 0 & $?$ \\
RT1247-1 & Baili & 0 & 8 & 0 & 7 & 6 & $?$ \\
RT847-3 & Weining & 0 & 1 & 0 & 1 & 0 & $?$ \\
RT1209-1 & Weining & 0 & 7 & 0 & 1 & 2 & $?$ \\
RT105-2 & Aigan & 0 & 1 & 1 & 1 & 8 & $?$ \\
RT194-4 & Aigan & 0 & 3 & 0 & 0 & 3 & $?$ \\
RT199-2 & Aigan & 0 & 1 & 0 & 0 & 0 & $?$ \\
RT304-3 & Aigan & 0 & 1 & 0 & 8 & 2 & $?$ \\
RT1163-2 & Aigan & 0 & 8 & 3 & 7 & 8 & $?$ \\
RT1200-1 & Aigan & 0 & 7 & 0 & 0 & 0 & $?$ \\
RT1220-6 & Aigan & 0 & 0 & 0 & 0 & 8 & $?$ \\
Mianyang11 & & 8 & 9 & 8 & 8 & 8 & $?$ \\
Rye Baili & & 0 & 0 & 0 & 0 & 0 & $?$ \\
Rye Weining & & 0 & 0 & 0 & 0 & 0 & $?$ \\
Rye Aigan & & 0 & 0 & 0 & 0 & 0 & $?$ \\
Chuannong10 & Aurora & 0 & 5 & 8 & 8 & 8 & $?$ \\
Chuannong11 & Aurora & 0 & 5 & 8 & 7 & 8 & $?$ \\
\hline
\end{tabular}

Infection types (IT) were scored based on a 0-9 scale, IT 0-3 were considered resistant, IT 4-6 were intermediate, and IT 7-9 were susceptible.

\section{Resistance analysis}

Five kinds of Pst pathotypes, CYR25 (European designation 15E156), CYR29, CYR30 (175E191) and CYR31 (239E175), CYR32, provided by the Plant Protection Institute, Sichuan Academy of Agricultural Sciences, were inoculated into seedlings at the two-leaf 
stage in a greenhouse. Infection types (IT) were scored based on a 0-9 scale, IT 0-3 were considered resistant, IT 4-6 were intermediate, and IT 7-9 were susceptible.

\section{RESULTS}

\section{Development of 12 new primary 1BL.1RS translocation lines}

From the progeny of $1 \mathrm{R}$ monosomic addition lines, which were derived from hybridized combinations between wheat cultivar MY11 and 3 rye varieties, 12 translocation lines were selected, in which 3 were derived from rye Baili, 2 derived from rye Weining, and 7 derived from rye Aigan (Table 1). Every translocation line was derived from a cross between single amphidiploid plant and its wheat parent MY11.

\section{Chromosome constitutions of the 12 new primary 1BL.1RS translocation lines}

To identify the chromosome constitutions of the 12 new primary translocation lines, GISH, Giemsa C-banding, and molecular markers were used.

The results of GISH (Figure 1) showed that all the 12 lines contained a pair of wheatrye translocation chromosomes. Yellow-green signals indicated rye chromosomes, while red signals indicated wheat chromosomes. However, GISH could not identify which chromosomes were involved in translocation. Giemsa C-banding was used further. According to the standard type of parents wheat and rye, Giemsa C-banding (Figure 2) proved that all the 12 translocation lines were wheat-rye 1BL.1RS translocation. Thus, the combination of GISH and Giemsa C-banding indicated that all the 12 lines $(2 \mathrm{~N}=42)$ were cytogenetically stable, containing a pair of 1BL.1RS translocation chromosomes.

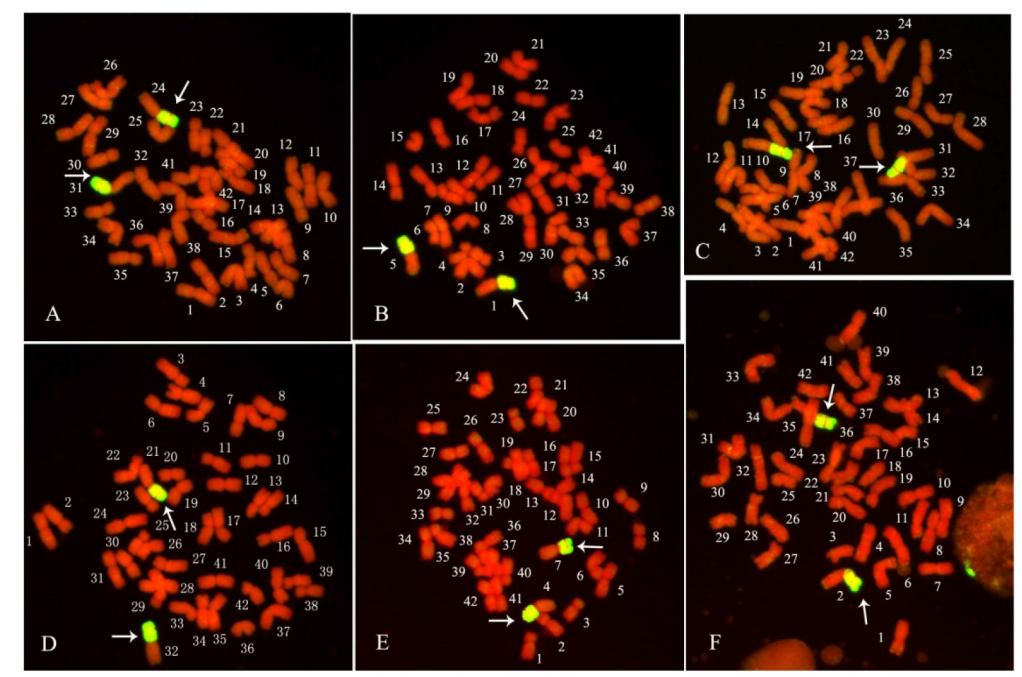

Figure 1. Genomic in situ hybridization of root tip chromosomes in wheat-rye translocation lines, which were originated from MY11 x rye. The rye genome DNA was labeled with digoxigenin-11-dUTP as probe, and the rye signal was detected with fluorescein-conjugated antidigoxigenin antibody. Yellow-green signals indicate rye chromatin. The arrows indicate wheat-rye translocated chromosomes. A. RT847-3; B. RT1209-1; C. RT1217-2; D. RT1235-1; E. RT1247-1; F. RT1200-1. 


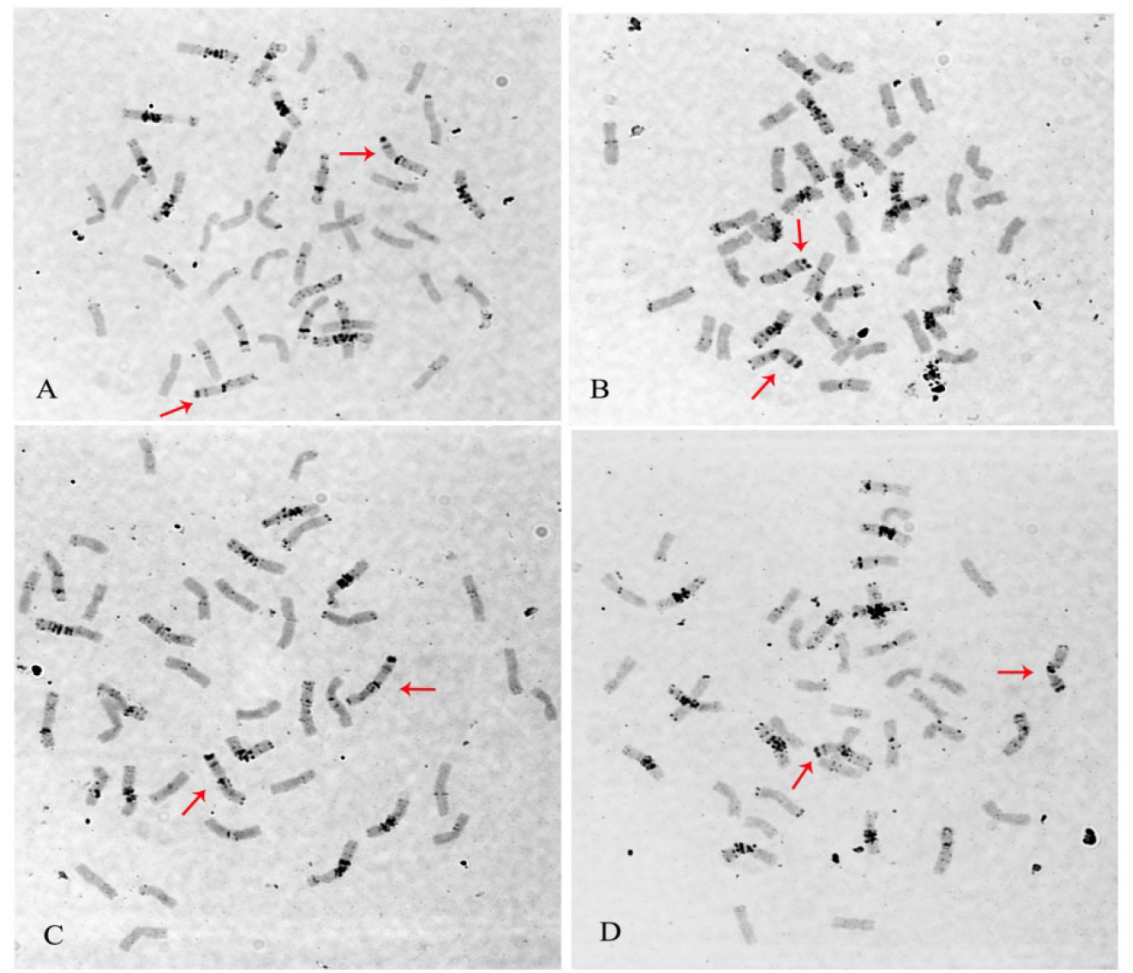

Figure 2. Giemsa C-banding of wheat-rye translocation lines. The arrows indicate the 1BL.1RS translocation chromosomes. A. RT199-2; B. RT304-3; C. RT105-2; D. RT194-4.

Primer pairs O11B3 and O11B5, $\omega$-sec-P1 and $\omega$-sec-P2 are the specific primers of 1BS and 1RS, respectively. Primer pairs O11B3 and O11B5 can amplify a 630-bp band on chromosome 1BS, while $\omega$-sec-P1 and $\omega$-sec-P2 amplify a 1076-bp band on chromosome 1RS. As shown in Figure 3, MY11 amplified a 630-bp band, while rye and all the translocation lines amplified a 1076-bp band. The PCR result showed that MY11 did not carry 1RS, but that the 12 translocation lines contained a pair of 1BL.1RS translocation chromosomes.

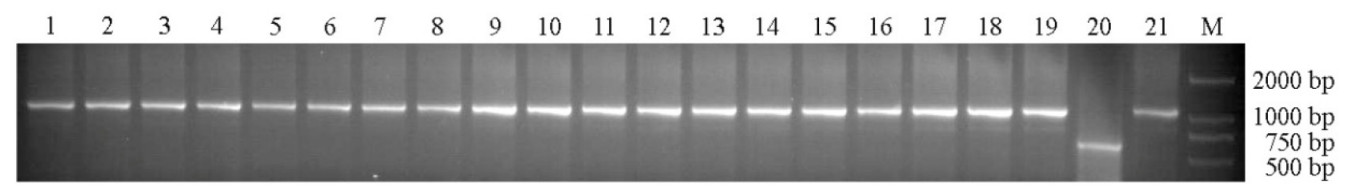

Figure 3. PCR result of four specific primers, O11B3 and O11B5, $\omega$-sec-P1 and $\omega$-sec-P2. Lane $1=\mathrm{RT} 1217$ 2; lane $2=\mathrm{RT} 1235-1$; lane $3=\mathrm{RT} 1247-1$; lane $4=\mathrm{RT} 847-3$; lane $5=\mathrm{RT} 1209-1$; lane $6=\mathrm{RT} 105-2 ;$ lane $7=$ RT194-4; lane 8= RT199-2; lane 9= RT304-3; lane 10=RT1163-2; lane 11=RT1200-1; lane 12= RT12206 ; lane $13=$ Chuannong10; lane $14=$ Chuannong11; lane $15=$ Chuannong 17; lane 16 = Chuannong18; lane $17=$ Rye Baili; lane 18 = Rye Weining; lane 19 = Rye Aigan; lane $20=$ Mianyang11; lane 21 = Rye L155 (Petkus); lane $M=$ marker. 
Primer PrCEN-2 was used to amplify a target fragment from the genomic DNA of all the translocation lines listed in Table 1. All the translocation lines and rye amplified an about 1000-bp band of rye centromere repetitive sequence, and no product was amplified from the DNA of MY11. The results (Figure 4) indicated that the primer PrCEN-2 was rye centromerespecific, and also indicated that all the 12 translocation lines contained the full $1 \mathrm{RS}$ arm of rye.

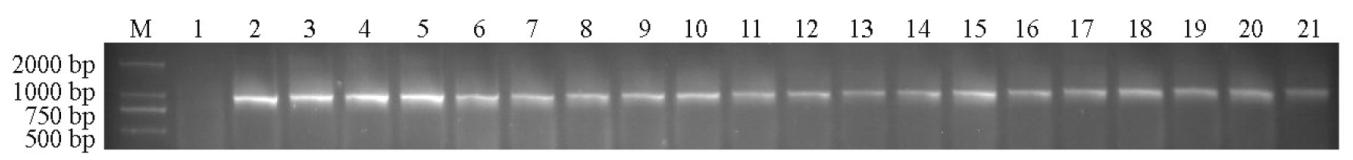

Figure 4. PCR result of primer PrCEN-2. Lane $M=$ marker; lane $1=\mathrm{MY} 11$; lane $2=$ Rye L155 (Petkus); lane $3=$ Rye Baili; lane $4=$ Rye Weining; lane $5=$ Rye Aigan; lane $6=$ RT1217-2; lane $7=$ RT1235-1; lane $8=$ RT12471; lane 9 = RT847-3; lane 10= RT1209-1; lane 11 = RT105-2; lane 12 = RT194-4; lane 13 = RT199-2; lane 14 = RT304-3; lane 15= RT1163-2; lane 16= RT1200-1; lane $17=\mathrm{RT} 1220-6$; lane $18=$ Chuannong10; lane $19=$ Chuannong11; lane 20= Chuannong17; lane 21= Chuannong18.

In conclusion, the 1BL.1RS chromosomes in these 12 primary translocation lines contain integrated 1RS chromosome arms, which originated from a different plant or pollen of rye.

\section{Analysis for resistance to stripe rust}

As shown in Table 1, wheat parent MY11 was highly susceptible to the five Pst pathotypes (CYR25, CYR29, CYR30, CYR31, and CYR32), while the three rye varieties were highly resistant. Wheat cultivars $\mathrm{CN} 10$ and $\mathrm{CN} 11$, in which their 1BL.1RS chromosomes came from the Russian wheat cultivar Aurora $(\mathrm{Yr}$ 9), were highly susceptible to CYR30, CYR31, and CYR32, but showed intermediate resistance to CYR29 and high resistance to CYR25. CN17 and $\mathrm{CN} 18$ showed resistance to five pathogens. The 1BL.1RS translocation lines derived from three Chinese local ryes were more resistant to stripe rust than those derived from Aurora. The 12 translocation lines exhibited five different phenotypes for resistance to infection by the five Pst pathotypes. Four lines showed high resistance to all five $P$ st pathotypes (phenotype 1). Three lines showed high resistance to Pst pathotypes CYR25, CYR30, CYR31, and CYR32, but were susceptible to CYR29 (phenotype 2). One line showed high resistance to Pst pathotypes CYR25, CYR29, CYR30, and CYR32, but was susceptible to CYR31 (phenotype 3). Two lines showed high resistance to Pst pathotypes CYR25, CYR29, CYR30, and CYR31, but were susceptible to CYR32 (phenotype 4). Two lines showed high resistance to Pst pathotypes CYR25, CYR 30, but were susceptible to CYR29, CYR31, and CYR32 (phenotype 5) (Table 1).

\section{DISCUSSION}

\section{Development of new wheat-rye translocations}

Translocation lines are important materials to study genetics, physiology and phytopathology, because an alien chromosome segment could be investigated in different genetic background. However, development of a large number of translocation lines would be very difficult. Ren et al. $(1990 \mathrm{a}, \mathrm{b})$ discovered that wheat chromosome pairing was disordered due to the presence of a single-rye chromosome in monosomic addition lines, leading to a high 
frequency of breakage and fusion between rye and wheat chromosomes during meiosis and producing Robertsonian translocations. In this study, we found that the high rate of translocation occurred in the offspring population of $1 \mathrm{R}$ monosomic wheat/rye addition lines. The plants contained the 1BL.1RS chromosome in the offspring population grown strongly in the field, and therefore, they were easily selected. Consequently, 12 new primary 1BL.1RS translocation lines were obtained and every one of them was derived from an independent offspring population of a single amphidiploid plant and their common wheat parent, a pure line isolated from cultivar MY11. Because rye varieties are outcrossing populations, these translocation lines were presumed to be genetically variable only with respect to their 1RS chromosomes.

\section{Identification of new translocations with the combination of molecular and cytological analysis}

Since large populations need to be screened to obtain translocations, a more reliable and easier means of identifying the alien chromatin is needed. Giemsa C-banding cannot identify the chromosome constitutions without obvious characteristic bands. GISH is considered an efficient method to identify alien chromatin introgression. However, GISH could not distinguish which alien chromatin has been transferred to the wheat background. The use of molecular markers was a fast way to indentify alien chromatin, but unstable. Up to now, many molecular markers have been developed, including the rye specific marker $\operatorname{Pr} 20 \mathrm{H}$, designed according to the rye-specific repetitive sequence of $\mathrm{pSc} 20 \mathrm{H}$ ( $\mathrm{Fu}$ et al., 2010), and specific markers of each rye chromosome arm (Xu et al., 2012; Tomita and Seno, 2012). Fu et al. (2010) considered that the combination of molecular and cytological analysis could increase the efficiency of detecting alien chromatin introgressions. In the present study, GISH, Giemsa $\mathrm{C}$-banding and molecular markers were used to identify the chromosome constitutions of the 12 new primary translocation lines. Cytological analysis showed that all the 12 lines contained a pair of 1BL.1RS translocation chromosomes. Primer pairs O11B3 and O11B5 and $\omega$-sec-P1 and $\omega$-sec-P2 are the frequently used markers to identify the presence of 1BS and 1RS. The PCR results of the specific primers of 1BS and 1RS proved the presence of 1RS and absence of 1BS. Rye centromere-specific primer PrCEN-2 was used to analyze the structure of the centromere of the 1BL.1RS chromosomes. As a result, all the 12 1BL.1RS lines contained rye centromere repetitive sequence. Altogether, these results clearly showed that every one of all the 12 translocation lines contained an integrated chromosome arm of 1RS, and that their genetic variation was not related to cytological deletion of their 1RS chromosome arms.

\section{Origin of diversity resistance in rye and its significance as alien species in genome modification of wheat}

The 1BL.1RS translocation from the Russian wheat cultivar Aurora, in which the 1 RS arm was derived from Petkus rye, has been the most widespread alien translocation in wheat-breeding programs, and hundreds of commercial cultivars from this translocation have been released (Rabinovich, 1998). However, as the sources of the 1BL.1RS translocation were limited, there are very few genetic variations in this 1 RS arm (Lelley et al., 2004). Since the 1980s, resistance genes $\operatorname{Yr} 9, \operatorname{Pm} 8, \operatorname{Lr} 26$, and $\mathrm{Sr} 31$ located on this 1RS arm, are no longer effective against new biotypes of the respective pathogens (Yang and Ren, 2001), and consequently, the frequency of 1BL.1RS translocation in recently released wheat cultivars has 
declined. However, rye (S. cereale) is a cross-pollinated plant, and the population of a variety is often genetically heterozygous. Different resistances to stripe rust were not only present in the 1BL.1RS translocations originating from different rye varieties, but also within the same rye variety. Ren et al. (2009) reported one novel resistant gene $\operatorname{YrCN17}$, which is located on the $1 \mathrm{RS}$ arm and likely to be an allele of $Y r 9$. Numerous variations could probably be expected in rye varieties (Ren et al., 2011a,b).

In the present study, 12 primary $1 \mathrm{BL} .1 \mathrm{RS}$ translocation lines, in which their 1RS arms were respectively derived from Baili, Weining and Aigan ryes, exhibited five different phenotypes for resistance to infection by the five Pst pathotypes, which are different from Chuannong10 and Chuannong11 with the gene $\operatorname{Yr} 9$ (Table 1). Because wheat parent MY11 was highly susceptible to the 5 Pst pathotypes, it is deduced that the resistance gene(s) in the 12 translocation lines must be located on these 1RS arms. Because all 12 translocation lines contain an integrated chromosome arm of $1 \mathrm{RS}$, their variation in resistance is only related to the genes on 1RS chromosome arms, maybe being alleles of the gene $\mathrm{Yr}$ 9. It is obvious that there are at least five different genes (alleles) for resistance to stripe rust in the 1RS arms from the populations of three rye varieties. In China, rye plants in nature cannot be infected by Puccinia striiformis f. sp Tritici. It is a very interesting question: how was the diversity of resistance genes (alleles) for wheat stripe rust in rye populations generated and maintained, which would not be subject to the selection pressure in plant-pathogen systems? It is undeniable that natural selection by parasites and interactions between hosts and parasites are expected to produce a positive response for generating and maintaining genetic diversity for resistance genes in natural populations (Tiffin and Moeller, 2006; Salvaudon et al., 2008). In the present study, the results suggest that the neutral diversity of resistance genes (alleles) for wheat stripe rust exists in the genome of an alien species, rye. These genes (alleles) would be generated by natural mutations and maintained in rye populations, which are an important genetic resource for wheat genome modification. These genes (alleles) would be functional in the wheat genome by developing wheat-rye translocation. It is very interesting how the genes (alleles) for resistance to wheat stripe rust from rye coevolved in rye, wheat and wheat-rye translocation lines, in which they are under selective pressures or not.

\section{ACKNOWLEDGMENTS}

Research supported by the National Natural Science Foundation of China (\#31271722).

\section{REFERENCES}

Anderson JA, Ogihara Y, Sorrells ME and Tanksley SD (1992). Development of a chromosomal arm map for wheat based on RFLP markers. Theor. Appl. Genet. 83: 1035-1043.

Berzonsky WA, Clements RL and Lafever HN (1991). Identification of 'Amigo' and 'Kavkaz' translocations in Ohio soft red winter wheats (Triticum aestivum L.). Theor. Appl. Genet. 81: 629-634.

Chai JF, Zhou RH, Jia JZ and Liu X (2006). Development and application of a new codomint PCR marker for detecting 1BL.1RS wheat-rye chromosome translocations. Plant Breed. 125: 302-304.

Conner RL, Thomas JB and Whelan EDP (1991). Comparison of mite resistance for control of wheat streak mosaic. Crop Sci. 31:315-318

Flor HH (1971). Current status of the gene-for-gene concept. Annu. Rev. Phytopathol. 9: 275-296.

Fu S, Tang Z, Ren Z and Zhang H (2010). Transfer to wheat (Triticum aestivum) of small chromosome segments from rye (Secale cereale) carrying disease resistance genes. J. Appl. Genet. 51: 115-121. 
Lelley T, Eder C and Grausgruber H (2004). Influence of 1BL.1RS wheat-rye chromosome translocation on genotype by environment interaction. J. Cereal Sci. 39: 313-320.

Liu J, Liu X, Dai L and Wang G (2007). Recent progress in elucidating the structure, function and evolution of disease resistance genes in plants. J. Genet. Genomics 34: 765-776.

Lukaszewski AJ, Lapinski B and Rybka K (2005). Limitations of in situ hybridization with total genomic DNA in routine screening for alien introgressions in wheat. Cytogenet Genome Res. 109: 373-377.

Lutz J, Limpert E, Bartos P and Zeller FJ (1992). Identification of powdery mildew resistance genes in common wheat (Triticum aestivum L.). Plant Breed. 108: 33-39.

Marais GF, Horn M and Toit FD (1994). Intergenetic transfer (rye to wheat) of a gene(s) for Russian wheat aphid resistance. Plant Breed. 113: 265-271.

Mater Y, Baenziger S, Gill K, Graybosch R, et al. (2004). Linkage mapping of powdery mildew and greenbug resistance genes on recombinant 1RS from 'Amigo' and 'Kavkaz' wheat-rye translocations of chromosome 1RS.1AL. Genome 47: 292-298.

Milus EA, Kristensen K and Hovmoller MS (2009). Evidence for increased aggressiveness in a recent widespread strain of Puccinia striiformis f. sp. tritici causing stripe rust of wheat. Phytopathology 99: 89-94.

Porter DR, Webster JA, Burton RL, Puterka GJ, et al. (1991). New sources of resistance to greenbug in wheat. Crop Sci. 31: 1502-1504.

Porter DR, Webster JA, Burton RL and Smith EL (1993). Registration of GRS1201 greenbug multi-biotype-resistant wheat germplasm. Crop Sci. 33: 1115.

Porter DR, Webster JA and Friebe B (1994). Inheritance of greenbug biotype G resistance in wheat. Crop Sci. 34: 625-628.

Rabinovich SV (1998). Importance of wheat-rye translocations for breeding modern cultivars of Triticum aestivum L. Euphytica 100: 323-340.

Ren Z and Zhang H (1997). Induction of small-segment-translocation between wheat and rye chromosomes. Sci. China C Life Sci. 40: 323-331.

Ren ZL, Lelley T and Röbbelen G (1990a). The use of monosomic rye addition lines for transferring rye chromatin into bread wheat. I. The occurrence of translocations. Plant Breed. 105: 257-264.

Ren ZL, Lelley T and Röbbelen G (1990b). The use of monosomic rye addition lines for transferring rye chromatin into bread wheat. II. The breeding value of homozygous wheat/rye translocations. Plant Breed. 105: 265-270.

Ren TH, Yang ZJ, Yan BJ, Zhang HQ, et al. (2009). Development and characterization of a new 1BL.1RS translocation line with resistance to stripe rust and powdery mildew of wheat. Euphytica 169: 207-213.

Ren TH, Chen F, Yan BJ, Zhang HQ, et al. (2011a). Genetic diversity of wheat-rye 1BL.1RS translocation lines derived from different wheat and rye sources. Euphytica 183: 133-146.

Ren TH, Chen F, Zou YT, Jia YH, et al. (2011b). Evolutionary trends of microsatellites during the speciation process and phylogenetic relationships within the genus Secale. Genome 54: 316-326.

Salvaudon L, Giraud T and Shykoff JA (2008). Genetic diversity in natural populations: a fundamental component of plant-microbe interactions. Curr. Opin. Plant Biol. 11: 135-143.

Sharma-Poudyal D and Chen XM (2011). Models for predicting potential yield loss of wheat caused by stripe rust in the U.S. Pacific Northwest. Phytopathology 101: 544-554.

Shepherd KW (1973). Homoeology of Wheat and Alien Chromosomes Controlling Endosperm Protein Phenotypes. In: Proceedings of the Fourth International Wheat Genetics Symposium (Sears ER and Sears LMS, eds.). Cytogenetics, Columbia, 745-760.

Shi ZX, Chen XM, Line RF, Leung H, et al. (2001). Development of resistance gene analog polymorphism markers for the Yr9 gene resistance to wheat stripe rust. Genome 44: 509-516.

Tiffin P and Moeller DA (2006). Molecular evolution of plant immune system genes. Trends Genet. 22: 662-670.

Tomita M and Seno A (2012). Rye chromosome-specific polymerase chain reaction products developed by primers designed from the EcoO109I recognition site. Genome 55: 370-382.

Van Campenhout S, Vander SJ, Sagi L and Volckaert G (1995). Locus-specific primers for LMW glutenin genes on each of the group 1 chromosomes of hexaploid wheat. Theor. Appl. Genet. 91: 313-319.

Villareal RL, Banuelos O, Mujeeb-Kazi A and Rajaram S (1998). Agronomic performance of chromosome 1B and T1BL.1RS near-isolines in the spring bread wheat Seri M82. Euphytica 103: 195-202.

Xu H, Yin D, Li L, Wang Q, et al. (2012). Development and application of EST-based markers specific for chromosome arms of rye (Secale cereale L.). Cytogenet. Genome Res. 136: 220-228.

Yang ZJ and Ren ZL (1997). Expression of gene Pm8 for resistance to powdery mildew in wheat for Sichuan. J. Sichuan Agric. Univ. 15: 452-456.

Yang ZJ and Ren ZL (2001). Chromosomal distribution and genetic expression of Lophopyrum elongatum (Host) A. Löve genes for adult plant resistance to stripe rust in wheat background. Genet. Resour. Crop. Evol. 48: 183-187. 
Zeller FJ and Fuchs E (1983). Cytology and disease resistance of 1A/1R and some 1B/1R wheat-rye translocation cultivars. Z. Pflanzenzecht 90: 285-296.

Zeller FJ and Hsam SLK (1983). Broadening the Genetic Variability of Cultivated Wheat by Utilizing Rye Chromatin. Proc. 6th Int. Wheat Genet. Symp., Kyoto, 161-173.

Zhang Y, Lubberstedt T and Xu M (2013). The genetic and molecular basis of plant resistance to pathogens. J. Genet. Genomics 40: 23-35.

Zhou Y, He ZH, Zhang GS and Xia LQ (2004). Utilization of 1BL/1RS translocation in wheat breeding in China. Acta Agron. Sin. 30: 531-535. 